\title{
Intrigues of immunogenetics in tuberculosis pathology and management-focused researches that could impact outcomes in Nigerian populations
}

\begin{abstract}
At the 2014 World Health Assembly, the World Health Organization's (WHO) End TB Strategy was approved and it calls for a $90 \%$ reduction in tuberculosis (TB) deaths and an $80 \%$ reduction in TB incidence rate by 2030 . According to the WHO's Global Tuberculosis Report of 2016, there were an estimated 10.4million new (incident) TB cases worldwide in 2015, people living with HIV accounted for 1.2 million $(11 \%)$ of all new TB cases. It also revealed that Nigeria and five other countries accounted for $60 \%$ of the new cases. Worldwide, the rate of decline in TB incidence remained at only $1.5 \%$ from 2014 to 2015 . This, according to the WHO, needs to accelerate to a $4-5 \%$ annual decline by 2020 to reach the first milestones of the End TB Strategy. Achieving this would require scaling up, easing and maximizing the various aspects of TB management including diagnostics, immunization, treatment, prevention services, social protection and other aspects of tuberculosis management particularly in Nigeria which ranks high among the WHO's high TB burden countries, high TB-HIV countries, and countries with high MDR-TB burden. One of the major challenges of TB control in Nigeria is difficulty in quickly and easily establishing reliable diagnosis. While tuberculosis smear microscopy is the gold standard diagnostic method, it has been associated with false negative results in $2-28.4 \%$ of cases by various studies. Furthermore, the duration of TB treatment ( 2 months for initial intensive phase and a 6-month continuation phase) has made treatment default to be a major challenge affecting up to $30 \%$ patients and most prominent $(78.1 \%)$ during the continuation phase, thus necessitating the need for newer shorter course treatments. Also, the inability of the currently available BCG vaccine to confer full protection throughout life is also driving research for new vaccines and biomarkers. Yet the poor understanding of the underlying mechanism of progression from latent to active tuberculosis disease state remains the biggest puzzle that had remained unsolved in tuberculosis research. The finding of a strong host genetic control of anti-mycobacterial immunity raises questions about the extent of the roles played by host genetics in tuberculosis phenotypes. This examines the sojourn of science towards demystifying the apparent interplay of immunology and genetics and how current trends seem to be edging closer towards solving some of the major questions in tuberculosis research that had eluded scientists for decades.
\end{abstract}

Keywords: Tuberculosis, Immunology, Genetics, Immunogenetics, Biomarkers, Gene expression
Volume 2 Issue 3 - 2017

\author{
Adepoju Paul Olusegun \\ Department of Medical Laboratory Science and Zoology, \\ Babcock University, Nigeria
}

Correspondence: Adepoju Paul Olusegun, Department of Medical Laboratory Science and Zoology, Babcock University, Nigeria, llishan-Remo, Ogun state, Nigeria,Tel +234 806088 7107,Email adepojupaul@gmail.com
Received: November II, 2017 | Published: November 28, 2017

\section{Introduction}

\section{Tuberculosis is a serious disease}

Tuberculosis remains one of the major global causes of mortality, accounting for about three million deaths per year. ${ }^{1}$ and regarded as the world's worst killer infectious disease. ${ }^{2}$ The disease outlook is further worsened by an increasing prevalence of multidrug-resistant strains of Mycobacterium tuberculosis, the global pandemic of HIV/AIDS (which increases susceptibility to tuberculosis) and the long period of chemotherapy required - combined they make tuberculosis control to be very difficult to achieve. Global targets and milestones for reductions in the burden of TB disease in the period 2016-2035 have been set as part of the Sustainable Development Goals (SDGs) and WHO's End TB Strategy. The first milestones of the End TB Strategy, set for 2020 , are a $35 \%$ reduction in the absolute number of TB deaths and a $20 \%$ reduction in the TB incidence rate, compared with levels in 2015. To reach these milestones, the TB incidence rate needs to be falling by $4-5 \%$ per year globally by 2020 and the proportion of people with TB who die from the disease (the case fatality ratio or CFR) needs to be reduced to $10 \%$ globally by 2020 .

\section{Discussion}

\section{TB immunology}

Since the main route of entry of the causative agent is the respiratory route, alveolar macrophages are the important cell types which combat the pathogen. ${ }^{3}$ Several studies have established that tuberculosis activates various aspects of macrophage-mycobacterium interactions; and macrophages play active roles in host response such as the binding of M. tuberculosis to macrophages via surface receptors. There are also very important immunological processes involved in the body's immune response to tuberculosis causative agent and the disease progress and the processes include phagosome-lysosome fusion, mycobacterial growth inhibition/killing through free radical- 
based mechanisms such as reactive oxygen and nitrogen intermediates; cytokine-mediated mechanisms; recruitment of accessory immune cells for local inflammatory response and presentation of antigens to $\mathrm{T}$ cells for development of acquired immunity. ${ }^{3-5}$ A number of studies ${ }^{6-8}$ have also been carried out on TB-specific acquired immune response through CD4 T cells - mainly responsible for protective Th1 cytokines and through CD8 cells bringing about cytotoxicity. But there is an incomplete understanding of the involvement-or extent - of CD-1 restricted CD8(+) Tcells and non-MHC restricted gamma/delta T cells as lamented by Raja. ${ }^{3}$ Since 1986, the presence of humoral immune response to TB infected has been reported ${ }^{9}$ although it remains not implicated in protection. Likewise, Barnes, et al., ${ }^{7}$ Denis, ${ }^{8}$ and others have reported the value of cytokine therapy to TB immunology even as the influence of the host human leucocyte antigens on the susceptibility to disease has also been extensively discussed by Selvaraj, et al. ${ }^{11}$

\section{TB genetics: organism and host}

Twin studies, ${ }^{12,13}$ animal models, ${ }^{14-17}$ then segregation analyses ${ }^{18,19}$ supported the hypothesis that there could be genetic susceptibility to TB in humans. There had also been numerous candidate gene studies, in addition to at least seven genome-wide linkage scans. ${ }^{20-26}$ But there is a great deal of inconsistency across these studies. Among studies of any candidate gene, there are always several reports that provide both positive and negative evidence for an association with tuberculosis.

\section{Sample, targets and biomarkers}

As Cliff, et al. ${ }^{27}$ rightly noted, the peripheral blood is easily accessible and is a good reservoir of immune cells moving to and from the sites of active disease and lymphoid organs. Cancer studies were the first to use peripheral blood to assess gene expression profiles in diseases; this was followed by autoimmune pathologies. On the overall, analyzing blood transcriptome has been of success in measuring the immune response to infection as reviewed by Blankley, et al. ${ }^{28}$ Berry, et al, ${ }^{29}$ Cliff, et al. ${ }^{30}$ and Bloom, et al.$^{31}$ successfully used pathway and modular bioinformatics analyses to characterize a transcriptional response to identify combinations of pathways and networks associated with tuberculosis. Gene expression studies have also revealed differences in the transcriptome between healthy controls and active TB or LTBI patients. ${ }^{32,33}$ Overall gene expression array results vary due to diverse genetic background of the study population and differences in the study design.

$\mathrm{Lu}$, et $\mathrm{al}^{34}$ conducted a gene expression microarray study to investigate the possibility of using mRNAs as biomarkers to differentiate active TB from LTBI. In their study, the expression of IFN- $\gamma$, the biomarker used in Interferon-Gamma Release Assays (IGRA), was not significantly different between the active TB and LTBI group. ${ }^{34}$ Instead, the combination of three genes, CXCL10 (chemokine $\mathrm{C}-\mathrm{X}-\mathrm{C}$ motif ligand 10), ATP10A (ATPase, class V, type 10A) and TLR6 (toll-like receptor 6) appeared to be effective at distinguishing between active and latent TB infection. In contrast, IL-8 (Interleukin 8), FOXP3 (forkhead box P3), and IL-12ß (interleukin 12 beta) were demonstrated to be the best discriminating biomarkers for TB and LTBI by Wu et al. ${ }^{35}$ Discrepancies between the two studies may be attributable to the differences in genetic background. At the same time, these findings suggest that not only are gene expression biomarkers more significant indicators of active TB, but they may also represent a more sensitive detection method for LTBI. Nevertheless, the same combination of genetic markers may not be applicable in another population. Lee and colleagues conducted a microarray experiment, comparing the gene expression profiles in the peripheral blood mononuclear cells among individuals with active TB, latent infection, and healthy conditions in a Taiwanese population. ${ }^{36}$ Bioinformatics analysis revealed that most of the differentially expressed genes belonged to immune responses, inflammation pathways, and cell cycle control. Subsequently, they carried out RT-PCR validation through which they identified four differentially expressed genes, NEMF, ASUN, DHX29, and PTPRC, as potential biomarkers for the detection of active and latent TB infections. Receiver operating characteristic analysis showed that the expression level of PTPRC may discriminate active TB patients from healthy individuals, while ASUN could differentiate between the latent state of TB infection and healthy condition. In contrast, DHX29 may be used to identify latently infected individuals among active TB patients or healthy individuals. To test the concept of using these biomarkers as diagnostic support, they constructed classification models using these candidate biomarkers and found the Naïve Bayes-based model built with ASUN, DHX29, and PTPRC to yield the best performance.

Evidence to date demonstrates that blood transcriptomics present a robust approach for studying the immune response in tuberculosis and other diseases, as similar findings have now been reported from various groups in different geographical locations. The findings offer information as to the immune response underlying the pathogenesis of tuberculosis and may provide tools toward diagnosis, treatment monitoring, and in the development of host-directed therapy regimens to support drug treatment. The potential use of blood transcriptomics in the clinical management of tuberculosis is likely to support current diagnostic tests and may help to speed up diagnosis and treatment, thus reducing transmission. The development of clinic-friendly tools for supporting the diagnosis of tuberculosis and treatment monitoring have been suggested to rely on a set of discriminant classifiers (consisting of between 12 and 100 genes) that would be easily convertible into a PCR-based affordable assay, allowing its development in the clinic for tuberculosis diagnosis.

\section{Tuberculosis: a global public health immunogenetic intrigue}

Epidemiological data suggesting that only 1 in $10 \mathrm{M}$. tuberculosisinfected persons will progress to clinical disease allow for an important effect of genetically determined host resistance factors ${ }^{37}$ and this why numerous candidate genes have been assessed in case-control studies of tuberculosis, and one of such studies investigated 17 candidate genes in a South African population. ${ }^{38}$ Truth be told, assessing the contribution(s) of host genetics to human tuberculosis has been a longstanding research challenge and evidence of genetic factors has come primarily from twin studies such as that of Puffer $^{39}$ and Kallmann, et al., ${ }^{13}$ and risks to first-degree relatives of cases. In addition, inferences of strong genetic influences have come from anecdotal accounts of socially prominent families, population variation in TB incidence and susceptibility to infection, and secular changes in TB severity, incidence and mortality inferred from historical information of contact between different populations,$^{40}$ as well as accidental inoculation of vaccinees with $M$. tuberculosis as documented by in The History of Tuberculosis by Daniel. ${ }^{41}$

\section{Is immunogenetics the missing piece in TB puzzle?}

A number of host genetic factors have been directly implicated in tuberculosis susceptibility but strong genetic effects on tuberculosis risk have been difficult to detect both by candidate gene and genomewide association studies. ${ }^{42,43}$ The reason for current inability to trace 
strong genetic effects is unknown although a number of possible explanations are supported by direct experimental data. For example, it has been shown that host genetic control of susceptibility is limited to specific host M. tuberculosis strain combinations. ${ }^{44}$ In addition, it is known that proper inclusion of gene environment interactions is of critical importance for the detection of strong host genetic effects on tuberculosis susceptibility. ${ }^{38}$ Most of the human genetics studies in tuberculosis have focused on the identification of genetic variants that impact on progression from infection to disease while only a few studies have attempted to identify genes that impact have direct on resistance to infection with $\mathrm{M}$. tuberculosis or genes that control the extent of anti-mycobacterial immunity.

\section{Immunity and genetics interplay}

Studies from South Africa, ${ }^{45}$ France $^{46}$ and Canada $^{40}$ focused on the study of innate resistance to infection with M. tuberculosis. Employing the tuberculin skin test as a tool to evaluate resistance to infection, a major locus (TST1) on chromosomal region 11p14 was identified that T-cell independent resistance to M. tuberculosis. In addition, a second major locus (TST2), on chromosomal region $5 \mathrm{p} 15$ was identified that controls the intensity of T-cell mediated delayed type hypersensitivity (DTH) to tuberculin. These results paved the way for the understanding of the molecular mechanisms involved in resistance to $M$. tuberculosis infection in endemic areas (TST1), and for the identification of critical regulators of T-cell dependent DTH to tuberculin (TST2) according to Schurr ${ }^{39}$ Furthermore, the finding of a strong host genetic control of anti-mycobacterial immunity raises questions about the extent of the role played by host genetics in other phases and stages of tuberculosis disease progression, particularly the progression from latent to active tuberculosis. Even though according to Schurr, ${ }^{39}$ immunologic models have been extensively proposed as the more realistic ways to predict which latently infected individual would end up with active tuberculosis, results of epidemiological studies in highly endemic areas and experiments in animal models that suggested a strong contribution of host genetic factors to tuberculosis vaccine efficacy make identification of the participating genes in active and latent tuberculosis to be one of the new frontiers of mycobacterial research.

\section{Conclusion}

As new methods emerge, tuberculosis researchers are able to answer more questions regarding the disease. However, isolation of immunological studies from genetics-based investigation had slowed down the pace of progress in the field of tuberculosis diagnosis, prevention and treatment. As more evidences emerge, it has gotten clearer that immunogenetics play critical roles in tuberculosis and approaches that put these evidences into consideration would prove critical in achieving the set SDG goals. But there is still another problem that immunogenetics may not be able to solve even though it can answer it - how can the population-based variations in Immunogenetics manifestations be harnessed to establish a global TB policy instead of population based outcomes that maybe difficult to implement elsewhere? This is the major dilemma and biggest future threats to countries like Nigeria that are worst hit but least prepared to combat TB using latest solutions.

\section{Acknowledgements}

None.

\section{Conflict of interest}

The author declares no conflict of interest.

\section{References}

1. World health organization. Global Tuberculosis Report. Switzerland: WHO; 2016. p. 12-35.

2. World health organization. Global Tuberculosis Report. Switzerland: WHO; 2017. p. 19-42.

3. Raja A. Immunology of tuberculosis. Indian JMed Res. 2004;120(4):213232.

4. Barnes PF, Abrams JS, Lu S, et al. Patterns of cytokine production by mycobacterium-reactive human $\mathrm{T}$-cell clones. Infect Immun. 1993;61(1):197-203.

5. Hart PD, Armstrong JA, Brown CA, et al. Ultrastructural study of the behavior of macrophages toward parasitic mycobacteria. Infect Immun. 1972;5(5):803-807.

6. Hoheisel G, Zheng L, Teschler H, et al. Increased soluble CD14 Levels in BAL fluid in pulmonary tuberculosis. Chest. 1995;108(6):1614-1616.

7. Barnes PF, Modlin RL, Ellner JJ. T-cell responses and cytokines. In: tuberculosis: pathogenesis, protection, and control (Bloom BR). Washington DC, USA: American Society for Microbiology; 1994. p. $417-418$.

8. Schlesinger LS, Kaufman TM, Iyer S, et al. Differences in mannose receptor-mediated uptake of lipoarabinomannan from virulent and attenuated strains of Mycobacterium tuberculosis by human macrophages. J Immunol. 1996;157(10):4568-4575.

9. Edwards D and Kirkpatrick $\mathrm{CH}$. The immunology of mycobacterial diseases1 2. Am Rev Resp Dis. 1986;134(5):1062-1071.

10. Denis M. Killing of Mycobacterium tuberculosis within human monocytes: activation by cytokines and calcitriol. Clinical \& Experimental Immunology. 1991;84(2):200-206.

11. Selvaraj P, Reetha AM, Uma H, et al. Influence of HLA-DR and-DQ phenotypes on tuberculin reactive status in pulmonary tuberculosis patients. Tubercle and Lung Disease. 1996;77(4):369-73.

12. Comstock GW. Epidemiology of Tuberculosis 1-3. American Review of Respiratory Disease. 1982;125(3P2):8-15.

13. Kallmann FJ, Reisner D. Twin studies on the significance of genetic factors in tuberculosis. American Review of Tuberculosis and Pulmonary Diseases. 1943;47(6):549-571.

14. Blackwell J, Barton C, White J, et al. Genomic organizaton and sequence of the human NRAMP gene: identification and mapping of a promotor region polymorphism. Mol Med. 2004;1(2):194-205.

15. Flynn J, Goldstein $\mathrm{M}$, Chan $\mathrm{J}$, et al. Tumor necrosis factor-alpha is required in the protective immune response against Mycobacterium tuberculosis in mice. Immunity. 1995;2(6):561-572.

16. Kramnik I, Dietrick W, Demant P, et al. Genetic control of resistance to experimental infection with virulent Mycobacterium tuberculosis. Proc Natl Acad Sci. 2000;97(15):8560-8565.

17. Lurie M. Heredity, constitution and tuberculosis: An experimental study. Am Rev Tuberc. 1941;44(3):1-125.

18. Shaw MA, Collins A, Peacock CS, et al. Evidence that genetic susceptibility to Mycobacterium tuberculosis in a Brazilian population is under oligogenic control: linkage study of the candidate genes NRAMP1 and TNFA. Tuberc Lung Dis. 1997;78(1):35-45.

19. Stein CM, Nshuti L, Chiunda AB, et al. Evidence for a major gene influence on tumor necrosis factor-alpha expression in tuberculosis: path and segregation analysis. Hum Hered. 2005;60(2):109-118.

20. Bellamy R, Beyers N, McAdam K, et al. Genetic susceptibility to tuberculosis in Africans: A genome-wide scan. Proc Natl Acad Sci. 2000;97(14):8005-8009. 
21. Jamieson S, Miller E, Black G, et al. Evidence for a cluster of genes on chromosome 17q11-q21 controlling susceptibility to tuberculosis and leprosy in Brazilians. Genes and Immunity. 2004;5(1):46-57.

22. Miller E, Jamieson S, Joberty C, et al. Genome-wide scans for leprosy and tuberculosis susceptibility genes in Brazilians. Genes Immun. 2004;5(1):63-67.

23. Cooke GS, Campbell SJ, Bennett S, et al. Mapping of a Novel Susceptibility Locus Suggests a Role for MC3R and CTSZ in Human Tuberculosis. Am J Respir Crit Care Med. 2008;178(2):203-207.

24. Stein CM, Zalwango S, Malone LL, et al. Genome scan of M. tuberculosis infection and disease in Ugandans. PLoS Onw. 2008;3(12):e4094.

25. Baghdadi JE, Orlova M, Alter A, et al. An autosomal dominant major gene confers predisposition to pulmonary tuberculosis in adults. $J$ Exp Med. 2006;203(7):1679-1684.

26. Mahasirimongkol S, Yanai H, Nishida N, et al. Genome-wide SNP-based linkage analysis of tuberculosis in Thais. Genes Immun. 2009;10(1):7783.

27. Cliff JM, Kaufmann SH, McShane H, et al. The human immune response to tuberculosis and its treatment: a view from the blood. Immunological reviews. 2015;264(1):88-102.

28. Blankley S, Berry MP, Graham CM, et al. The application of transcriptional blood signatures to enhance our understanding of the host response to infection: the example of tuberculosis. Phil Trans $R$ Soc B. 2014;369(1645):20130427

29. Berry MP.An interferon-inducible neutrophil-driven blood transcriptional signature in human tuberculosis. Nature. 2010;466:973-977.

30. Cliff JM, Lee JS, Constantinou N, et al. Distinct phases of blood gene expression pattern through tuberculosis treatment reflect modulation of the humoral immune response. J Infect Dis. 2013;207(1):18-29.

31. Bloom CI, Graham CM, Berry MP, et al. Detectable changes in the blood transcriptome are present after two weeks of antituberculosis therapy. PLoS One. 2012;7(10):e46191.

32. Constantoulakis P, Filiou E, Rovina N, et al. In vivo expression of innate immunity markers in patients with Mycobacterium tuberculosis infection. BMC infectious diseases. 2010;10(1):243.

33. Jacobson KR, Tierney DB, Jeon CY, et al. Treatment outcomes among patients with extensively drug-resistant tuberculosis: systematic review and meta-analysis. Clin Infect Dis. 2010;51(1):6-14.
34. Lu C, Wu J, Wang $\mathrm{H}$, et al. Novel biomarkers distinguishing active tuberculosis from latent infection identified by gene expression profile of peripheral blood mononuclear cells. PloS One. 2011;6(8):e24290.

35. Wu B, Huang C, Kato-Maeda M, Hopewell PC, et al. Messenger RNA expression of IL-8, FOXP3, and IL-12 $\beta$ differentiates latent tuberculosis infection from disease. J Immunol. 2007;178(6):3688-3694.

36. Lee PH, Fu H, Lai TC, et al. Glycemic control and the risk of tuberculosis: a cohort study. PLoS Med. 2016;13(8):e1002072.

37. Posey DL, Naughton MP, Willacy EA, et al. Implementation of new TB screening requirements for US-bound immigrants and refugees 2007 2014. MMWR Morb Mortal Wkly Rep. 2014;63(11):234-246.

38. Möller M, De Wit E, Hoal EG. Past, present and future directions in human genetic susceptibility to tuberculosis. FEMS Immunol Med Microbiol. 2010;58(1):3-26.

39. Puffer RR. Familial susceptibility to tuberculosis. Its importance as a public health problem. Familial susceptibility to tuberculosis. Its importance as a public health problem. 1994;39(4):107-108.

40. Schurr E. The contribution of host genetics to tuberculosis pathogenesis Kekkaku. 2011;86(1):17-28.

41. Daniel TM. The history of tuberculosis. Respiratory medicine 2006;100(11):1862-1870.

42. Thye T, Vannberg FO, Wong SH, et al. Genome-wide association analyses identifies a susceptibility locus for tuberculosis on chromosome 18q11. 2. Nat Genet. 2010;42(9):739-741.

43. Di Pietrantonio T, Correa JA, Orlova M, et al. Joint effects of host genetic background and mycobacterial pathogen on susceptibility to infection. Infection and Immunity. 2011;79(6):2372-2378.

44. De Wit E, Van der Merwe L, Van Helden PD, et al. Gene-gene interaction between tuberculosis candidate genes in a South African population. Mammalian Genome. 2011;22(1-2):100-110.

45. Van Crevel R, Ottenhoff TH, Van der Meer JW. Innate immunity to Mycobacterium tuberculosis. Clin Microbiol Rev. 2002;15(2):294-309.

46. Memari B, Bouttier M, Dimitrov V, et al. Engagement of the Aryl Hydrocarbon Receptor in Mycobacterium tuberculosis-Infected Macrophages Has Pleiotropic Effects on Innate Immune Signaling. $J$ Immunol. 2015;195(9):4479-4491. 